\title{
Is there a role for intraoperative radiation therapy in resectable extremity soft tissue sarcoma beyond external beam radiation therapy?
}

\author{
Interview with Antonino De Paoli ${ }^{1}$ and Claudia Sangalli ${ }^{2}$ by Giacomo G. Baldi ${ }^{3}$
}

In favor:

Antonino De Paoli'

Against:

Claudia Sangalli ${ }^{2}$

\section{Introduction}

Surgery with negative margins is the primary and only curative treatment in localised extremity soft tissue sarcoma (ESTS). One of the most important concepts in modern oncology is to focus local treatment not only on achieving the best local control, but also on the preservation of functionality and quality of life of patients $[1,2]$.

Within such approaches, less extensive surgery is often required in specific anatomical locations, which provides improved functional outcome, but with the necessity of additional local treatment modalities to maintain adequate local control, usually by the use of external beam radiation therapy (EBRT).

However, in some situations, adequate doses of radiotherapy (RT) cannot be applied without severe side effects to the surrounding normal tissue, and in such situations, the application of a single dose of intraoperative radiotherapy (IORT) during the surgical procedure might be beneficial. The use of an IORT boost would not only result in smaller postoperative treatment volumes but may also allow the possibility of excluding organs at risk (major nerves or skin for ESTS) from the radiation field, which could re-

\footnotetext{
'Department of Radiation Oncology, CRO-IRCCS,

National Cancer Institute, Aviano, Italy.

${ }^{2}$ Department of Radiology and Radiotherapy,

Fondazione IRCCS Istituto Nazionale dei Tumori, Milan, Italy.

3"Sandro Pitigliani" Department of Medical Oncology,

Hospital of Prato, Prato, Italy.

Correspondence to:

Giacomo G. Baldi,

"Sandro Pitigliani" Department of Medical Oncology,

Hospital of Prato,

Via Suor Niccolina 20, 59100 Prato, Italy.

Phone: +39 0574802525 - Fax: +390574 802503

E-mail: giacomogiulio.baldi@uslcentro.toscana.it

CANCER BREAKING NEWS 2017;5(1):12-14

DOI: $10.19156 / \mathrm{cbn} .2017 .0033$
}

duce late toxicities and improve long-term functional outcome. If applied before postoperative EBRT, an increased wound complication rate might be avoided. Consequently, this would combine the advantage of the postoperative approach with the smaller treatment volumes that, from the preoperative setting, are known to be beneficial $[3,4]$.

Nevertheless, this technique has some major limitations. For example, IORT is available only at a small number of referral centres and most of the evidence base comes from rather small retrospective analyses with similarly short follow-up and patient cohorts that are not homogeneous. In this interview, two opinion leaders in this field from two Italian sarcoma referral centres attempt to clarify the role of IORT beyond EBRT in radically resected ESTS.

\section{What do you think about the impact on critical surgical margins of IORT rather than EBRT in ESTS? Might it be more useful than EBRT for local control in selected cases?}

\section{Pro IORT (as part of combined modality treatment)}

IORT should be considered as a component of a combined modality treatment with EBRT ( \pm chemotherapy) rather than as an alternative to EBRT in ESTS. IORT is a nonconventional treatment modality that allows a radiation dose escalation program at surgery, targeting the high risk area (critical surgical margins) after tumor resection. This dose escalation (e.g., boost) may complete the preoperative or postoperative EBRT in the limb-sparing approach of ESTS, combining conservative surgery and radiation therapy. Its use as a single modality rather than EBRT could be considered only in selected patients with recurrent disease, previously treated with EBRT, in attempting a conservative surgical salvage (see below).

\section{Against IORT (pro EBRT plus limb-sparing surgery)} EBRT combined with limb-sparing surgery local control rates are comparable to those achieved with amputation [5]. The benefit of adding EBRT to limb-sparing surgery has 
been addressed in two randomized trials; both showed that combined treatment reduced the risk of local recurrence by $20-25 \%$ when compared to limb-sparing surgery alone [6, 7]. Thus, the use of adjuvant EBRT maximizes functional and cancer outcomes without the significant morbidity and cosmetic deformity of radical surgery [7]. Margin status has been reported to be the most important prognostic factor for local control even in patients treated with combined surgery and radiotherapy. Total escalated doses of radiotherapy that can be delivered even with the most sophisticated and updated EBRT precision techniques could be limited by the presence of dose-limiting surrounding organs or structures in planning the treatment volume [8].

Intraoperative electron-beam radiation therapy (IOERT) is considered as a component of treatment; this modality has the advantage of delivering a high boost dose to deep-seated sarcoma residues or risk surgical bed areas adjacent to radiosensitive critical organs by mobilizing these structures temporarily out of the radiation field [9].

The question is that you do not know the margins status prior to the dose delivery. This may result in overtreatment or on the other hand lead to inappropriate treatment for both dose and for volumes.

2. Do you think that there may be a positive impact on acute and late toxicities, functional outcome and limb preservation rates with the use of IORT rather than EBRT in ESTS?

\section{Single modality IORT not recommended}

Only a few experiences of the use of IORT alone rather than EBRT with conservative surgery in ESTS are available. These experiences include old, small series of patients, with poor functional results because of the large treatment volumes and the high single dose required. On the basis of these experiences, the use of IORT as a single modality rather than EBRT in ESTS should not be recommended [10].

\section{Against IORT}

Currently a preoperative radiation treatment is preferred in the management of soft tissue sarcoma. In fact preoperative treatment, by reducing treatment volumes and doses, has led to a reduction in side effects mainly due to lower exposure to skin and nerve structures.

Moreover I think that IORT could not improve functional outcome and limb preservation rates.

Besides this, although a randomized study that compared preoperative versus postoperative RT showed no difference in terms of local recurrence rate [11], there is some evidence from retrospective studies to suggest that the effects of close or positive margins on local relapse may be minimized with preoperative RT $[12,13]$. A study from Toronto showed that the use of a radiation boost (IORT or EBRT) in ESTS following preoperative $\mathrm{RT}$ and resection with positive margins did not enhance local control [13].

\section{In some retrospective series with IORT there was a slight increase of severe postoperative wound complications and infections. Do you think that it may be a limitation for the improvement of this technique in the near future?}

\section{Pro IORT}

I do not think so. In those retrospective series, IORT was correctly combined with EBRT and postoperative wound complications were mainly related to the EBRT component, in particular when preoperative EBRT was used. One of the major advantages of IORT is the possibility of excluding the skin, subcutaneous and muscle-uninvolved tissues from the radiation field, minimizing the influence on wound healing. More recently reported experiences with the use of preoperative EBRT with modern RT techniques show more favourable results in terms of complications, and IORT boost remains a very favourable option when critical, positive or close surgical margins are expected. Preoperative EBRT and IORT boost should be considered in particular when reconstructive surgery with flaps is needed, because of the possibility of sparing the vulnerable reconstruction and avoiding postoperative EBRT [14].

\section{Against IORT}

I think that the use of electron beam or brachytherapy as an IORT approach does not change the risk of side effects such as wound complications, neuropathy or skin injury. The literature reports that patients with more intense acute side effects experienced long-term side effects more frequently and intensely if treated by IORT [15].

The major risk of side effects has been found in patients affected by lower extremities soft tissue sarcoma. In my experience, I don't think that technological improvement could decrease the risk of toxicities.

\section{Who, if one exists, is the ideal patient that could be a candidate for IORT rather than EBRT only?}

\section{Pro IORT}

In ESTS, IORT should be given in combination with EBRT rather than as its alternative. IORT as a single RT 
modality could be considered only in selected patients with recurrent disease, previously treated with EBRT, in an attempt at conservative surgical salvage.

\section{Against IORT}

I would propose IORT alone as an adjuvant approach in elderly patients. In this way, it is possible to avoid logistical problems, such as the difficulty of undergoing radiation treatment every day.

\section{Despite the lack of prospective randomized data, do you routinely use IORT in your referral centre for selected patients with ESTS?}

\section{Pro IORT}

Although randomized trials comparing EBRT + IORT versus $\mathrm{EBRT}$ alone are not available, retrospective series and the more recent pooled analyses report encouraging results on local control with excellent rates of preserved limb function $[4,16]$. Our institutional approach will likely

\section{References}

1. Kretzler A, Molls M, Gradinger R et al. Intraoperative radiotherapy of soft tissue sarcomas of the extremity. Strahlenther Onkol 2004;180:365-70.

2. Oertel S, Treiber M, Zahlten-Hinguranage A et al. Intraoperative electron boost radiation followed by moderate doses of external beam radiotherapy in limb-sparing treatment of patients with extremity soft-tissue sarcoma. Int J Radiat Oncol Biol Phys 2006;64:1416-23.

3. Roeder F, Lehner B, Schmitt $T$ et al. Excellent local control with IOERT and postoperative EBRT in high grade extremity sarcoma: results from a subgroup analysis of a prospective trial. BMC Cancer 2014;14:350.

4. Roeder F, Lehner B, Saleh-Ebrahimi L et al. Intraoperative electron radiation therapy combined with external beam radiation therapy and limb sparing surgery in extremity soft tissue sarcoma: a retrospective single center analysis of 183 cases. Radiother Oncol 2016;119:22-9.

5. Rosenberg SA, Tepper J, Glatstein E et al. The treatment of soft-tissue sarcomas of the extremities: prospective randomized evaluations of (1) limb-sparing surgery plus radiation therapy compared with amputation and (2) the role of adjuvant chemotherapy. Ann Surg 1982;196:305-15.

6. Pisters PW, Harrison LB, Leung DH et al. Long-term results of a prospective randomized trial of adjuvant brachytherapy in soft tissue sarcoma. J Clin Oncol 1996;14:859-69.

7. Yang JC, Chang AE, Baker AR et al. Randomized prospective study of the benefit of adjuvant radiation therapy in the treatment of soft tissue sarcomas of the extremity. J Clin Oncol 1998;16:197-203.

8. Delaney Trust Fund. Radiation therapy: neoadjuvant, adjuvant, or not at all. Surg Oncol Clin N Am 2012;21:215-41. continue to include IORT after preoperative EBRT \pm chemotherapy in selected patients for limb-sparing surgery in ESTS when close or positive margins of resection are expected.

\section{Against IORT}

Because of the lack of pathological data, the risk of complications and because of the good results in terms of local control with preoperative EBRT with or without chemotherapy, I would not propose IORT to our patients in our referral centre.

\section{Acknowledgments}

The authors thank Ray Hill, an independent medical writer, who provided native English editing and journal styling on behalf of HPS. This editorial assistance was funded by PharmaMar, Spain.

\section{Conflicts of Interest}

The Authors declare there are no conflicts of interest in relation to this article.

9. Gunderson LL, Willet CG, Calvo FA, Harrison LB. Intraoperative irradiation techniques and results. Current Clinical Oncology. Springer Science \& Business Media; Second edition 2011.

10. Azinovic I, Martinez-Monge R, Javier Aristu J et al. Intraoperative radiotherapy electron boost followed by moderate doses of external beam radiotherapy in resected soft tissue sarcomas of the extremities. Radiother Oncol 2003;67:331-7.

11. O'Sullivan B, Davis AM, Turcotte R et al. Preoperative versus postoperative radiotherapy in soft-tissue sarcoma of the limbs. A randomised trial. Lancet 2002:359:2235-41.

12. Dagan R, Indelicato DJ, McGee L et al. The significance of a marginal excision after preoperative radiation therapy for soft tissue sarcoma of the extremity. Cancer 2012;118:3199-207.

13. Al Yami A, Griffin AM, Ferguson PC et al. Positive surgical margins in soft tissue sarcoma treated with preoperative radiation: Is a postoperative boost necessary? Int J Radiat Oncol Biol Phys 2010;77:1191-97.

14. O'Sullivan B, Griffin AM, Dickie CL et al. Phase II study of preoperative image guided intensity modulate radiation therapy to reduce wound and combined modality morbidities in lower extremity soft tissue sarcoma. Cancer 2013;119:1878-94.

15. Niewald M, Fleckenstein J, Licht $\mathrm{N}$ et al. Intraoperative radiotherapy (IORT) combined with external beam radiotherapy (EBRT) for soft-tissue sarcomas - a retrospective evaluation of the Homburg experience in the years 1995-2007. Radiat Oncol 2009;4:32

16. Roeder F, De Paoli A, Saleh-Ebrahimi L et al. Intraoperative electron radiation therapy after gross total resection in extremity soft tissue sarcoma: a pooled analysis. Radiother Oncol, in press. 